\title{
Comunicación moral e instituciones intermediarias en las sociedades modernas ${ }^{1}$
}

\author{
Thomas Luckmann
}

Universität Konstanz

Edición y traducción a cargo de Rita Radl. Universidad de Santiago

\section{Resumen}

En el presente texto, el autor trata el tema de la comunicación moral en las sociedades modernas. Apoyándose en una amplia muestra de datos procedentes de un gran proyecto de investigación subvencionado por el Consejo Científico Alemán (DFG), muestra que en las sociedadcs modernas las orientaciones morales siguen teniendo especial relevancia, existe una moral en uso, que se difunde específicamente en las instituciones intermediarias tales como la familia, los peer groups, grupos cívicos y religiosos, asociaciones, etc. Argumenta la hipótesis que el estilo dominante de la comunicación moral en las sociedades modernas es de tipo indirecto.

Palabras clave: comunicación moral, instituciones intermediarias, moral en uso, moralización indirecta y directa.

\section{Abstract. Moral communication and intermediary institutions in modern societies}

In this article, the author tries the theme of moral comunication in modern societies. It starts from a broad sweep rescarch data about moral communication in Germany it was sponsored by the German Science Fundation. The rescarch data seems that morality is in use all arround us and it is transmitted in a specifically way by intermediary institutions like the family, peer groups, civic and religious organizations, associations, etc.

1. Éste es el texto inédito de la conferencia magistralmente pronunciada por el profesor Thomas Luckmann, el día 15 de mayo de 2000 en las facultades de Filosofía y Ciencias de la Educación de la Universidad de Santiago, acto organizado por la profesora Rita Radl Philipp a través del Departamento de Sociología y Ciencia Política y de la Administración.

Algunos aspectos de esta versión editada de la conferencia, pronunciada en el mes de mayo de 2000 en la Universidad de Santiago de Compostela, ya han sido desarrollados en ponencias anteriores en la Universidad de Erlangen, Viena, Salzburgo, en el Congreso de la Asociación Nórdica de Semiótica de Imatra (Finlandia) en 1996 y en la Universidad de Bergen. Asimismo, una versión posterior ha llegado a ser publicada como lección pública $\mathrm{n}^{\circ} 17$ por el Collegium Budapest/Institute of Advanced Studies, en marzo de 1997, bajo el título: «The moral order of Modern Societies, Moral Communication and Indirect Moralizing». Véase, asimismo: LuCKMARM, TH. (1996). "Über Moral und moralische Kommunikation in der modernen Gesellschaft». En: Sociologia Internationalis, 34/1, Berlín, p. 1-11. 
Add the hypothesis of sorts about the dominant style of moral communication in modern societies; the preferred style in modern life is an indirect style of moralizing.

Key words: moral communication, intermediary institutions, morality in use, indirect and direct moralizing.

\section{Sumario}

$\begin{aligned} \text { 1. Introducción } & \text { 4. La moralidad-en-uso } \\ \text { 2. El argumento anticipado } & \text { 5. Estilos de moralización } \\ \text { 3. La disolución del orden moral } & \text { Anexo } \\ \text { tradicional } & \end{aligned}$

\section{Introducción}

Para investigar la moral en las sociedados tradicionales uno tiene que observar los preceptos éticos y los códigos morales establecidos, culturalmente objetivizados e institucionalmente asegurados, y, desde luego, uno tiene que averiguar la manera en que los miembros de la sociedad moralizan. En la sociedad moderna, sin embargo, observar la moral en códigos escritos y en los roles institucionalizados de la conducta llega a ser un cometido en vano. En la sociedad moderna la manera de investigar la moral es atendiendo a la comunicación moral. Trataré de explicar por qué eso es así.

No daré una definición precisa y exhaustiva de los términos, pero no se puede considerar innecesario indicar de modo general qué es lo que quiero decir cuando hablo de moralidad y moral y lo que entiendo por comunica ción y comunicación moral.

La moralidad es un grupo de nociones razonablemente coherentes de lo que está bien y lo que está mal, nociones de la vida buena que guían la acción humana más allá de la gratificación inmediata de los deseos y las exigencias momentáneas de una situación. Dichas nociones, como todas las nociones, las mantienen los individuos, pero no se originan en el individuo. Están construidas intersubjetivamente en la interacción comunicativa, y están seleccionadas, mantenidas y transmitidas en procesos sociales complejos. A lo largo de generaciones llegan a formar tradiciones históricas distinguidas en las que se articula una particular visión de la vida buena. Esto significa que algunos conceptos de lo que está bien y de lo que está mal están canonizados y otros, censurados. Una vez que se haya trazado el camino para llegar a ese ideal, se han establecido las bases del orden moral de una sociedad. El seguir ese camino se define como el ideal de la vida, y el ideal sirve como norma en la organización de la vida colectiva. Cuando se castigan sistemáticamente las desviaciones graves de la norma, es cuando el orden moral de la sociedad se establece por completo. 
Cuando hablo de comunicación, restrinjo el uso del término a los procesos basados en sistemas de signos socialmente construidos, particularmente, y no exclusivamente, el lenguaje. Los procesos son (inter)acciones sociales especiales. Son esenciales para la organización de la vida colectiva humana; son especialmente importantes por hacer posible la transmisión efectiva de las tradiciones de una sociedad, incluyendo aquéllas de su orden moral. Los procesos comunicativos son recíprocos o unilaterales; son directos, cara a cara, o mediados de múltiples maneras. Tienen lugar entre individuos como individuos o como detentores de un cargo, y como representantes de grupos o categorías socialmente definidas. De la misma manera, los individuos se dirigen a individuos o cargos, grupos y categorías socialmente definidas de individuos. Es más, la comunicación puede existir entre remitentes anónimos y destinatarios igualmente anónimos.

En cuanto a la comunicación moral, existe una distinción obvia entre argumentación moral y moralización. La argumentación podría estar, pero raramente está, libre de moralización, y la moralización no la necesita pero a veces emplea la argumentación. La argumentación va de declaraciones descriptivas sobre valores morales — aquí y allá- y la narración de ejemplos explícitos de moral de conducta a formulaciones abstractas de principios éticos y de criterios. La moralización puede ser positiva, como en las alabanzas, o negativa, como en las condenas, y puede consistir en las evaluaciones del comportamiento de otros y de las propias acciones de uno.

Los destinatarios de la comunicación moral podrían ser, asimismo, objetos de moralización, como cuando le riñen a un niño, o podrían ser los receptores de la moralización sobre otros, como en el caso de aquéllos que escuchan chismes. Los métodos para moralizar podrían ser lingüísticos en el sentido limitado del término: semántico-léxico, prosódico o retórico. Junto a éstos, o por sí solos, están los elementos paralingüísticos, miméticos o de gesticulación, que podrían transmitir asuntos moralmente significativos. Es más, ciertos géneros que podrían tener o no una función moral primaria, por ejemplo: máximas y proverbios, podrían utilizarse como componentes a modo de fórmulas de la comunicación moralizadora.

Finalmente, el estilo general de la moralización varía: puede ser directa, en forma de alabanza o queja, acusación, indignación, etc., o puede ser indirecta en forma de litotes, preguntas, formulaciones causales, ciertas maneras de tomar el pelo, etc.

Ahora vuelvo sobre la discusión acerca de la moralidad en las sociedades modernas. Comenzaré anticipando las conclusiones, es decir, resumiré la conclusión de mi argumento y después lo desarrollaré paso a paso. El conocimiento de la conclusión facilita el seguimiento de los pasos que llevan a la misma desde el principio. Primero resumiré los puntos de vista bien conocidos sobre la moralidad en la teoría sociológica clásica. A continuación resaltaré algunos de los problemas inherentes a estas opiniones y mostraré cómo considero que se pueden resolver. Finalmente, añadiré una hipótesis especí- 
fica sobre el estilo dominante de la comunicación moral en las sociedades modernas ${ }^{2}$.

\section{El argumento anticipado}

Según las teorías sociológicas modernas, la moralidad desapareció en gran parte de la estructura social de las sociedades modernas. Se dice que sus grandes instituciones dominantes, la economía y el Estado, siguen unas normas funcionales de hecho, aunque no siempre se emanciparon del orden moral tradicional de igual forma en su retórica autolegitimadora. Ya no requieren la justificación de un universo transcendente. Una vez que la opinión de que la moralidad estaba eliminada de la estructura social moderna se convirtió en parte del consenso sociológico, sólo faltaba un pequeño paso para otra suposición. No sólo se daba por hecho que la moral no tenía lugar en la organización racional-funcional de la estructura social, sino que se daba por supuesto de que ésta había desaparecido de la sociedad en general.

El diagnóstico principal es esencialmente correcto, pero la correspondiente suposición es errónea. La moralidad sigue prevaleciendo en las diversas interacciones sociales en las que nos vemos envueltos diariamente. Es posible que no sea el tipo de moralidad que las autoridades morales tradicionales desearían que tuviéramos, pero se trata de una visión razonablemente coherente de la vida buena. En vez de «moralidad» quizás habría que decir «moralidad o moralidades». El singular prejuzga un tema empírico que tan sólo puede resolverse analizando variadas formas de comunicación moral.

Al final especularé y haré la sugerencia de que en la sociedad moderna el estilo dominante de comunicación moral demuestra una preferencia por la moralización indirecta. Es una especulación porque la evidencia que apoya esta noción no es tan sistemática como la evidencia a favor de la presencia continuada de la moralidad en forma de moralización. Pero es una especulación controlada, porque existen evidencias a su favor. Junto con unas buenas bases teóricas que la apoyan, tiene un alto grado de verosimilitud.

Así pues, éstos serán los pasos de mi argumentación. Ahora me gustaría comenzar situando en una perspectiva histórica las razones por las cuales acepto la suposición sociológica clásica de que la moralidad ha desaparecido en gran parte de la estructura social de las sociedades modernas y después, las razones por las que rechazo la aceptación de la subsiguiente suposición de que la moralidad, en consecuencia, haya desaparecido por completo de la sociedad.

2. Una versión anterior de parte de este argumento se publicó con el título: «Privatization of Religion and Morals», en Paul HeElas, Scott LaSH y Paul MORRIS (eds.). Detraditio nalization. Critical Reflections on Authority and ldentity, Oxford, 1996, p. 72-86. 


\section{La disolución del orden moral tradicional}

Simplificando, se podría decir que en las sociedades arcaicas la religión, la moralidad y la ley (en tanto que se pueda hablar de la ley en ausencia de un código escrito) tenían una base común en la estructura social. Por lo menos, las instituciones que implicaban diferentes funciones en la vida social estaban muy coordinadas - y esto se refiere a aquellas instituciones con funciones religiosas, morales y legales. El concepto de la vida buena que está en el corazón del orden moral de toda sociedad estaba claramente articulado. Ese concepto estaba especificado en una amplia gama de comportamientos en cuanto a lo que se podía hacer y no hacer. Debido a que la vida seguía su curso en pequeñas comunidades, la violación del orden moral era claramente visible, y también su castigo. La autoridad sostenía que el orden moral estaba legitimado a través de la referencia sistemática a un universo trascendente sagrado. Éste fue incluso el caso cuando la moralidad y la religión ya no se consideraban lo mismo. La orientación, incluyendo la orientación moral en dicho mundo, fácil o no, era relativamente simple.

Es obvio que en las sociedades modernas un orden moral de este tipo ya no existe. Ni siquiera se aproxima a sus características. El transcurso de la transformación estructural que lleva a la aparición de la sociedad moderna es conocido; la disolución de la moral tradicional forma parte de esta transformación. Lo voy a explicar de forma escueta.

En el curso de la historia del cercano oriente y occidente, las funciones morales, religiosas y legales de la vida colectiva se organizaron cada vez más en instituciones especializadas y algo separadas que se organizaban sistemáticamente para llevar a cabo un particular grupo de funciones muy relacionadas entre sí. El codificar las reglas de conducta y de mala conducta en forma de ley, como por ejemplo en el Código de Hammurabi, es un caso a considerar. Aunque no se atribuían exclusivamente a una fuente divina, como por ejemplo: los Diez Mandamientos, dichos códigos (aún) no eran completamente independientes del universo sagrado. La ley permaneció sagrada hasta más o menos la promulgación del Código postrevolucionario de Napoleón. Durante todo este período, las instituciones religiosas continuaban siendo la sede oficial de la moral.

A medida que la diferenciación funcional de los cometidos políticos, económicos y legales de la vida social que marcaron las sociedades occidentales desde finales de la edad media se aceleraba, ni la religión ni el orden moral estaban exentos de este proceso general. Pero, incluso después de la aparición del estado y de la economía como territorios semiautónomos, las instituciones religiosas permanecieron como la base socioestructural del orden moral durante mucho tiempo. Pero como las iglesias también estaban sujetas a la especialización institucional, lo que se consideraba como su territorio propio se restringió cada vez más. Perdieron su papel en el gobierno y en la economía; lo que quedó fue la familia y el individuo. 
La disciplina social y moral ejercitada por las instituciones religiosas disminuyó rápidamente. Las complejas transformaciones culturales y estructurales a las que ponemos las etiquetas simplificadoras de Ilustración y Revolución Industrial, aceleraron el proceso, salvando temporalmente la periferia de la modernización. La religión y la moral eran cada vez más individualizadas y, primero en el caso de la religión y después (e incluso resultando más contradictorio en cuanto a su función esencial) en el caso de la moral, privatizadas. La religión y la moral miraron hacia dentro. Ya que habían perdido su sede socioestructural en las grandes instituciones públicas, mantuvieron durante un tiempo su influencia sobre la subjetividad individual. La religión se transformó en fe privada, y la moralidad, en conciencia subjetiva.

Hace por lo menos un siglo, Durkheim pensó que si la solidaridad orgánica - como él llamaba al orden moral necesario para las sociedades con una división compleja de trabajo- no llenaba el vacío dejado por el declive del orden moral tradicional asociado a sociedades más simples, la sociedad moderna llegaría a una anomia peligrosa. Manteniendo su convicción de que una sociedad sin un orden moral básico no podría sobrevivir, Durkheim buscó soluciones en lo que hoy algunos llamarían "cultura cívica», otros, «instituciones intermedias» y otros, "capital social».

Casi cincuenta años más tarde, Theodor Geiger, un importante sociólogo del derecho, empezó con un diagnóstico similar. Sin embargo, sus conclusiones fueron bastante diferentes. Él también dio por hecho que en las sociedades modernas el orden tradicional, bastante homogéneo y obligatorio, se estaba disolviendo. Pero a su juicio esto era una condición necesaria para la evolución de la sociedad moderna. Según su criterio, algunas de aquellas partes de la moralidad tradicional que no se habían transformado en ley positiva, solo podrían sobrevivir si se convertían en algo socialmente irrelevante $y$, para utilizar su término, espiritualizado.

\section{La moralidad-en-uso}

La opinión de Durkheim y Geiger sobre el hecho de que las sociedades modernas ya no poseen un orden moral general obligatorio la comparten la mayoría de los científicos sociales contemporáneos. No cabe duda de que debe aceptarse como esencialmente correcta. Sin embargo, las conclusiones de Durkheim y Geiger sobre las consecuencias de este hecho son completamente diferentes.

Durkheim probablemente erró al postular que ninguna sociedad, incluso la compleja sociedad moderna, podría existir sin la fuerza integradora de un código moral específico y al mismo tiempo de forma general obligatoria. $\mathrm{Al}$ contrario, tiene más sentido suponer, al igual que Geiger, que la organización racional de instituciones diferenciadas en las estructuras sociales modernas no sólo podría existir sin dicho orden moral, sino que éste sólo podría ser impedido por aquél. Utilizando la metáfora de Geiger, uno podría coincidir con él en que la moralidad se retiró de la estructura social. Se podría también 
aceptar su noción del tipo de moralidad que podría sobrevivir mejor en una situación en la cual la misma tiene que sobrevivir sin un hogar institucional y en la que la mayor parte de lo que puedes hacer y de lo que no puedes hacer se ha codificado en un sistema de ley positiva, de un tipo de Gesinnung sethlk, es decir, de una ética de disposición subjetiva y de una motivación en vez de una ética tradicional de responsabilidad y obligación. Sin embargo, Geiger se equivocó en otro asunto importante. No sólo han sobrevivido algunos elementos de la moralidad tradicional. Esto quizás se podría explicar en términos de residuo/fósil. Pero la afirmación de Geiger de que sea cual sea la moralidad que haya permanecido, ésta se haya evaporado en el aire raro de una espiritualidad pura, es, claramente, un error.

Otra posibilidad de contemplar el destino de la moral en el mundo moderno es bastante más probable. La moralidad siempre había sido una dimensión constitutiva de las interacciones sociales concretas cara a cara. Penetró en lo que Goffman llama el «orden de la interacción» en sociedades arcaicas en las que no había ninguna base institucional propia, como era el caso de las sociedades tradicionales. ¿No podría ser que después de la pérdida de la sede institucional de la religión-moral que ocupaba en la sociedad tradicional, la moralidad haya mantenido su hogar permanente al aire libre, es decir, en el orden de la interacción?

Después de todo, la moral en uso - como me gustaría llamar a la moral interactiva, practicada en pequeñas comunidades cara a cara - llegó primero. Sólo bajo ciertas condiciones sociales, caracterizadas por una división compleja del trabajo y del conocimiento, por la evolución del sistema de escritura, etc., surgieron históricamente instituciones morales más elaboradas. En las civilizaciones antiguas las nociones e ideas moralmente significativas se unían a sistemas complejos de moralidad. Éstos tenían sus cánones y catequismos y se introducían en las normas institucionales de la estructura social. Finalmente, desarrollaron una base estructural propia a través de las instituciones religiosomorales. Evidentemente, la aparición de esta superestructura cultural y base organizadora no hizo que la moralidad práctica desapareciese de la vida cotidiana de la sociedad, aunque ejerció una influencia definitiva sobre la dimensión moral de la interacción social situacional. El grado de influencia varió, dependiendo de la verosimilitud y el éxito de la difusión de una elevada retórica moral y un vocabulario moral de motivos, y dependiendo del grado del cumplimiento institucional de dogmas y catequismos morales.

En las sociedades modernas, órdenes morales homogéneos e unitarios de este tipo ya no se encuentran incrustados en grandes instituciones dominantes. Las nociones del bien y del mal, de lo correcto y lo incorrecto, sin embargo, continúan siendo relevantes para la conducta en la vida y, concretamente, para la planificación, ejecución y evaluación de las propias acciones y de las de los demás. Aunque una jerarquía dogmática de valores que contiene conceptos canónicos de la vida buena ya no se transmite uniformemente ni obligatoriamente por parte de un aparato institucional, algunas nociones de lo correcto y lo incorrecto aún se siguen transmitiendo a través de varios 
canales. Entre ellos las instituciones intermediarias son los más importantes: la familia, los peer-groups, las ramas locales de grupos más grandes de la socie$\mathrm{dad}$, asociaciones e instituciones tales como las organizaciones cívicas, los clubes, las congregaciones religiosas, y las escuelas, seminarios y academias. Las instituciones intermediarias son, así, la fuente principal de al menos una obligación parcial de la moral mencionada en el orden de la interacción. Esta parte del orden de la interacción la «localizamos» en los ambientes sociales y en las instituciones intermediarias de diferentes maneras.

Cuando nos quejamos de los demás o les acusamos de un delito u otro, cuando pedimos perdón por nuestras propias acciones y fallos, cuando nos indignamos e invitamos a los demás a que se unan a nuestra indignación, cuando alabamos y condenamos, cuando pronunciamos máximas y citamos proverbios, cuando facilitamos o buscamos consejo, y cuando chismorreamos, sermoneamos y decimos palabrotas, ejercitamos la comunicación moral explícita o implicita. Por lo tanto, continuamente demostramos a los demás, al igual que los demás nos demuestran, que algún tipo de moral se continúa practicando en todas partes.

Si es preciso disponer de pruebas por encima y mas allá de nuestra intuición y observación como miembros practicantes de nuestras sociedades para llegar a la conclusión de que las profecías del juicio final concernientes a un declive general y la destrucción de la moral en la sociedad moderna son erróneas, entonces los datos conseguidos en una serie de investigaciones que durante muchos años he realizado con mis colegas y ayudantes, lo comprueban ampliamente ${ }^{3}$. La moral se usa en todas partes. Sin embargo, por otra

3. Quizás convenga explicar la naturaleza de los datos. Consisten en cientos de horas de grabaciones de charlas de sobremesa de familias del sur y del este de Alemania de historias de conversión secular y religiosa, de chismes en entornos informales e institucionales, de llamadas de emergencia a los bomberos, de sesiones profesionales de consejos familiares, sexuales y genéticos, de entrevistas de admisión en hospitales psiquiátricos, de varios tipos de programas de radio con llamadas de los oyentes, de reuniones de grupos ecologistas locales, de debates públicos (sobre la Guerra del Golfo, por ejemplo) en directo y en televisión, de una serie de programas religiosos en la televisión, de una campaña antitabaco regional, de discursos públicos (por ejemplo el pronunciado por el presidente de la República Federal de Alemania en fin de año, y por varias figuras públicas en el cincuenta aniversario de la liberación de Auschwitz), etc.

Sin embargo, deberíamos mencionar algunas limitaciones. Con la excepción de algumas transcripciones americanas y japonesas, nos restringimos a Alemania, el bulto de los datos es del Sur. Las charlas de sobremesa de familias, una fuente importante para nuestro análisis de formas y géneros en la comunicación cara a cara, provienen sólo de las clases media-baja y media, y ninguna familia tenia niños pequeños. La validez de la afirmación de que la moral-en-uso está extendida en la sociedad moderna no tiene duda. Sin embargo, las preguntas sobre la distribución de estilos y repertorios de comunicación moral y, correspondientemente, las preguntas sobre las diferencias y las similitudes del sentido moral, no se pueden contestar con certeza incluso en el caso de Alemania, y las afirmaciones sobre otras sociedades estructuralmente comparables deben, por el momento, permanecer como especulativas. 
parte, no se puede negar um profundo cambio. La evidencia - ya anticipada en la teoría sociológica clásica - apoya la opinión de que una moral única y uniforme no persiste en la sociedad moderna.

Nosotros y otros seguimos normas morales diferentes. Si esta moral diversificada contiene algo así como una gramática moral profunda, es una pregunta abierta. Los resultados sobre este particular son incompletos; lo que tenemos es a menudo contradictorio.

Quizás debería añadir que en un principio mis colegas y yo dedicamos nuestros esfuerzos a la descripción y al análisis de las formas más importantes utilizadas en los procesos concretos de la comunicación moral. Pensamos que un enfoque cercano a las realidades de la vida cotidiana, nos conduciría a los aspectos sustantivos de la moralidad moderna. De hecho, encontramos muchas formas, formatos y géneros de comunicación moral, y aprendimos mucho sobre el sentido moral articulado en estas formas. Vimos el contorno del repertorio de la comunicación moral en la sociedad alemana contemporánea. La mayor parte, aunque no todas, de las características observadas probablemente encontraría su analogía en otras sociedades comparables ${ }^{4}$. Más tarde, a base de los resultados de las investigaciones anteriores y algunas adicionales, algunos de nosotros estudiaban más específicamente las instituciones intermediarias en las que se practica alguna forma de moralidad $^{5}$.

\section{Estilos de moralización}

En la introducción he distinguido entre moralización positiva y negativa, y entre moralización directa e indirecta. Las formas comunicativas y los géneros en los que se lleva a cabo una moralización bastante directa en situaciones cara a cara incluyen los chismes, las quejas, las secuencias de indignación conjuntas, al igual que análogos dialogísticos de sermones morales. En los medios de comunicación, desde los libros hasta la radio y la televisión, algunas de las formas y los modos de comunicación moral cara a cara se utilizan con ciertas modificaciones. Algunos programas sirven como foro de indignación pública, otros se ocupan de quejas y otros se quedan con los chismes. Es más, han surgido algunos géneros nuevos en los medios de comunicación con funciones moralizadoras, como es el caso de una restringida variante alemana de evangelismo televisivo condensado, y de seculares sermones morales por parte de personas en altos cargos que se supone tienen una autoridad moral.

4. Las investigaciones fueron patrocinadas por la Fundación Científica Alemana y dirigidas por Jörg BERGMANM y el que suscribe. Cf. Jörg BERGMAnN, Thomas LuCKMANn y otros, Die kommunikahwe Konstruktion der Moral, I y II, Opladen, 1999, 2000.

5. El estudio fue apoyado por la Fundación Bertelsmann. Cf. Moral im Alltag Sinnvermit tlung und moralische Kommunikation in intermediären Institutionen, Gütersloh, 1998. Mis asociados fueron Jörg Bergmann, Gabriela Christmann, Michaela Goll, Angela Keppler, Hubert KnOblauch y Ska WiltscheK. 
Aunque ni siquiera puedo emprender la tarea de resumir los resultados de estas investigaciones, me gustaría seleccionar unos cuanto ejemplos. Todos apoyan el claro presupuesto general de que no existe una institución moral central, y también el presupuesto menos obvio de que hay mucha moralidad práctica por todas partes, y que esta moral-en-uso está compuesta de varios tipos diferentes.

Así, por ejemplo, la familia. Algunos tipos de familia continúan transmitiendo la moralidad tradicional, el tipo dominante; sin embargo, se abstienen de propagar normas morales autoritarias y específicas, recalcando la autonomía del individuo. Las consultas que proliferan en las sociedades modernas con distribuciones sociales del conocimiento complejas demuestran una similitud formal con la familia tipo dominante: recalcan la selección individual y tienden a rechazar cualquier fuente transindividual de autoridad moral $^{6}$. Esto prevalece para gran parte de las consultas familiares y sexuales no relacionadas con la Iglesia. Es razonable suponer que la familia y el aparato de consultas representan un ajuste algo pasivo a la situación pluralista que caracteriza a la mayoría de las sociedades modernas. Sin embargo, los valores morales específicos se propagan no sólo a través de (algunas) entidades religiosas tradicionales, sino también a través de movimientos sociales de diferentes tipos. Entre los más recientes en Alemania está el "movimiento» ecológico, que, con sus morales escatológicas y ascéticas y su actividad misionera, es un caso con éxito en este punto.

Aunque haya que tener presente la naturaleza insatisfactoria de la evidencia sobre las siguientes observaciones, existen buenos motivos para suponer que durante las últimas generaciones se ha producido un cambio en el estilo preferente de moralización. A pesar de que no exista un orden general obligatorio y razonablemente uniforme en las sociedades modernas, todavía existe un set de moral-en-uso. La conducta de los individuos pertenecientes a variados grupos y ambientes continúa siendo evaluada por otros de acuerdo con alguna concepción de lo que está bien o lo que está mal. Sin embargo, fuera de los grupos y espacios de casa, hay bastante incertidumbre sobre si los criterios de evaluación son los compartidos por otros.

Las diferencias en las orientaciones morales existían ya en las sociedadcs tradicionales con un orden moral bastante homogéneo. Probablemente había alguna incertidumbre interactiva, más específicamente moral y moralizadora, en todas las comunidades arcaicas con excepción de las más simples. Sin embargo, la estructura de la interacción social en las comunidades pequeñas de la sociedad tradicional minimizaban esa incertidumbre. En las formas más complejas de interacción social en las antiguas civilizacioncs tradicionales, sobre todo en sus segmentos urbanos, los individuos que no se conocían, o que no se conocían bien, no podían evitar el riesgo de la incertidumbre

6. Esta formulación ha sido tomada de la contribución de Jörg BERGMANN, Michaela GOLL y Ska WiLTSCHEK, elaborada para el volumen de la Fundacion Bertelsmann arriba mencionado. 
moral. Cuando surgían situaciones en las que tenían que interactuar con extraños por cualquier motivo, el riesgo podía mantenerse en un nivel bajo, mientras uno podía percibir al otro bajo categorías sociales bien definidas y abiertamente marcadas. En las sociedades tradicionales estas categorías aportaban mucha información sobre el estatus moral de una persona. Naturalmente, cuando se trataba de desviaciones morales, actuales o potenciales, uno podía inclinarse o verse obligado a aceptar el riesgo del conflicto moral.

Por el contrario, en la vida plural moderna, la incertidumbre interactiva, moral y moralizadora es alta fuera del grupo familiar o del entomo del individuo. En las sociedades con un orden obligatorio y uniforme, la homogeneidad moral entre individuos se podría suponer, a no ser que se produjeran pruebas concretas que demostrasen lo contrario en la interacción. Se podría decir que en las sociedades modernas se da el caso contrapuesto. La homogeneidad moral no se da por sentada hasta que no se haya producido una evidencia positiva de la misma. En términos de Schutz: «La idealización de la congruencia de los sistemas de relevancia, una parte constitutiva de la tesis general de la reciprocidad de perspectivas, está patas arriba ${ }^{7}$.

Cuando la interacción no está restringida a los roles sociales estrechamente funcionales, altamente anónimos, es posiblemente útil o incluso esencial un cierto grado de homogeneidad moral para su realización. Invitaciones a indignaciones conjuntas, chismes, etc., son inherentemente arriesgados para personas que no se conocen bien. Evidentemente, bajo estas condiciones, la forma específica de la interacción comunicativa, que corre el riesgo fuerte de una incertidumbre moral y de un conflicto potencial, es la moralización directa. Si las cosas siguen igual, este aspecto de la vida moderna probablemente fomentará una fuerte preferencia por un estilo indirecto de comunicación moral. Dicho estilo será la preferencia en toda comunicación fuera del ámbito familiar de los individuos. Es más, se podría especular que bajo ciertas condiciones este estilo se difundirá en la comunicación moral, incluso en los ámbitos familiares.

Deberíamos recordar que la moralización indirecta no surgió primeramente en la sociedad moderna. Parece que en varios tipos de sociedades no modernas surgió este estilo bajo condiciones especiales y sólo en ciertos ámbitos, y se limitaba a pocas situaciones comunicativas. Por otra parte, en las sociedades modemas, parece que han surgido más «interámbitos» de comunicación y, como acabo de especular, no es del todo improbable que este estilo se haya extendido más allá del ámbito de la interacción en el cual su adopción había sido favorecida por causas estructurales de moralización mucho mas «intraámbito».

También se debería recordar que la conjetura sobre el predominio de la moralización indirecta no implica que en las sociedades modernas nadie

7. Cf. Alfred Schultz, "Common Sense amd the Scientific Interpretation of Human Action». En Collected Papers, I (ed. de Maurice Natanson). La Haya, 1962, p. 11f. y "Symbol, Reality and Society», op. cit., p. $315 \mathrm{f}$. 
moralice directamente. Es probable que en los grupos y ámbitos en los que la homogeneidad moral por parte de los participantes en la interacción comunicativa puede ser asumida, no existan motivos para preferir la moralización indirecta. Nuestros hallazgos apoyan esta suposición. Dicho estilo sólo es utilizado si se difunde en estos grupos o ámbitos por otros motivos. Existen pruebas de ello en la comunicación en la familia e incluso en el programa religioso de televisión que investigamos en nuestro estudio ${ }^{8}$. Sin embargo, todavía no tenemos el amplio barrido de datos necesario para establecer los repertorios de los diferentes ámbitos comunicativos en relación con el predominio del estilo directo o indirecto de moralización. No obstante, el contorno principal de la situación aparece.

En las sociedades no modernas las familias eran comunidades de vida en las cuales prevalecía un grado bastante alto de homogeneidad moral o se podía decir que prevalecía en aquéllas con una posición de autoridad. En la sociedad moderna éste ya no es el caso, y mientras una cierta cantidad de moralización directa tiene lugar donde prevalecen las relaciones asimétricas (ej. entre adultos y niños muy jóvenes) y entre los que comparten las mismas características y los que se conocen bien, generalmente se puede decir que se prefiere la moralización indirecta.

Las comunidades de vida como la familia ya no son por más tiempo necesariamente comunidades de personas que piensan igual (Gesinnungsge meinschaften ${ }^{9}$ ). Al mismo tiempo, diversos tipos de estas comunidades proliferan en las sociedades modernas. Los riesgos de interacción del tipo antes descrito no existen en ellas. Tan sólo en relaciones externas la homogeneidad moral no puede ser supuesta. Ahí, los identificados con las comunidades ideológico-morales tienen dos opciones básicas. O bien adoptan el estilo indirecto para permanecer moralmente indefinidos, o bien aceptan el riesgo y moralizan directamente, con lo cual inevitablemente se convierten en líderes morales. Sin embargo, la selección genuina entre las opciones sólo se abre para los miembros de Gesinnangsgemeinschaften cuando éstos se encuentran solos en su interacción con no miembros. Es posible que en las actividades colectivas externas de las comunidades ideológico-morales, sobre todo en aquéllas en las que domina un tipo de proselitismo, la opción de permanecer indefinido no es viable. El grupo podría esperar y exigir testimonio.

Otro aspecto relevante acerca de la situación en las sociedades modernas es que las empresas morales a menudo no se presentan como tales. Parece ser que sólo los tipos más tradicionales de Gesinnungsgemeinschaften, en especial aquéllas de persuasión fundamentalista, están todavía dispuestas a declararse. Vale la pena mencionar que muchas de las comunidades ideológico-morales más recientes siguen una estrategia diferente. Muchas guardan apariencias científicas, médicas, terapéuticas, etc. A nivel estructural, podría considerarse esto una forma indirecta.

8. Por Ruth AYALß.

9. «Comunidades de ideología compartida», nota de la traductora. 


\section{Anexo:}

Ejemplos de moralización indirecta, aquí traducidos, que corresponden a datos de investigación alemanes.

\section{Construcciones "por qué» (LA OPERADORA)}

S. llama a información y pregunta por el número de teléfono de una familia que se llama «Weisser» en Constanza.

12 A: No tengo ninguna familia WEISSER en Constanza,

13 sólo una familia WEISS.

14 S: Sí, pienso que viven en Reichenau y no directamente en Constanza.

16 A: [bruscamente] ¿Entonces POR QUÉ dijo Constanza?

17 S: Perdone, pensé que el Reichenau pertenecía a Constanza.

$18 \quad(2.5)$

19 A: Bueno, pues entonces el número es...

\section{Construcciones «no entiendo» (ANTJE Y PAUL)}

1 A: Bueno, bien, pero no te lleva a ninguna parte si tu...

2 P: $[j a, j a]$

3 No me llevará a ninguna parte, pero...

4 A: Si piensas ahora sólo tienes que ver primero

4 cómo puedes poner tus cosas en orden;

5 yo, éh!, no entiendo, no entiendo tampoco

6 por qué no llamas al tío ese en Stuttgart

7 y le dices ahora cuando hagas tu semestre de prácticas

8 que te gustaría - el próximo semestre-continuar

$9 \quad$ en Stuttgart otra vez.

\section{Reconstrucciones (ejemplo directo/indirecto)}

(ESTUDIANTE CHINO Y PROFESOR DE ALEMÁN)

$86 \mathrm{Hu}$ : Entonces el secretario de aquel partido en esos tiempos si

87 S:

[mm]

88 Hu: Se enfrentó a mi

89 S:

$[m m][\ldots]$

$97 \mathrm{Hu}$ : Otras personas entonces, querían

98 enfrentarse a mí. POR QUÉ no ACTÚAS 
99 como los demás, como tus compañeros de clase 100 vestirte tan bien.

102 S: [lleno de indignación] Oh! sí!

\section{Litotes (NOSOTROS LOS ALEMANES)}

$42 \mathrm{H}$ : Nosotros los Alemanes tienes que mirarlo de esta manera: a veces

43 no nos comportamos siempre justo de la manera correcta

44 no está siempre muy bien.

\section{Construcciones generalmente indirectas (CONSULTA GENÉTICA)}

23 KM: Nos gustaría tener hijos a nosotros,

24 de hecho nos gustan los niños: a mí me gustan y a mi mujer.

25 B: Creo, bueno, de hecho me gustaría.

26 KM: Creo.

27 B: No veo motivo por el que uno quería por ese motivo.

28 KM: Ejem.

29 B: Sin hijos.

30 KM: Ejem. (7.0) fue de hecho interesante que ellos...

Otras formas de ser indirecto y oblicuo

Eufemismos, falta de fluidez, comienzos falsos, reformulaciones, modulaciones humoristas, herramientas prosódicas: tono de queja, tono brusco, etc., con palabras semánticamente neutrales y no moralizadoras. 